\title{
NEW DATA ON A NUCLEAR SUBSHELLS OBTAINED FROM THE ANALYSIS OF THE INFORMATION FROM THE INTERNATIONAL DATABASE ON NUCLEAR STRUCTURE ENSDF
}

\author{
I.N.Boboshin ${ }^{1}$, B.S.Ishkhanov ${ }^{1,2}$, V.V.Varlamov ${ }^{1}$ \\ ${ }^{1}$ Skobeltsyn Institute of Nuclear Physics and \\ ${ }^{2}$ Physics Faculty of Lomonosov Moscow State University
}

\begin{abstract}
At last time a large amount of the experimental nuclear spectroscopy data was obtained and stored in large and complete databanks and databases. This is a good progress really but new very serious problem appears. Because of significant data systematical errors one is often forced to deal with very discrepant data and it is difficult to obtain reliable information from such data. To solve this problem and to remove the systematic errors, new technologies of the work with such kind data were developed. Using these new technologies one can obtain information with high accuracy and reliability, and in many cases, new information have not (could not) been obtained experimentally. Below such approach is presented concern nuclear spectroscopy data.
\end{abstract}

\section{INTRODUCTION: DATABASE ENSDF}

Modern large and complete databanks give to one the real possibilities to solve the problem mentioned above. They allow to overview data obtained in various times and using different methods, to choice the best data, to reveal systematical errors, to investigate their reasons and, often, to understand possible ways of data improving.

The oldest and biggest large and complete and really the best databank on nuclear spectroscopy is the ENSDF (Evaluated Nuclear Structure Data File) /1/ maintained by the USA National Nuclear Data Center (BNL, Brookhaven) on behalf of the international Nuclear Data Network sponsored by the International Atomic Energy Agency (Vienna, Austria). This file contains nuclear structure and decay data for all known nuclides. ENSDF consists (Fig. 1) of "data sets" including the evaluated values for properties of the levels and gamma-rays for all nuclei (Adopted Levels, Gammas data sets) and the "best values" for properties of the levels, gamma-rays and decays for each nucleus obtained from various types of experiments ("experimental" data sets). In each data set there is brief abstract containing description of the method of presented data obtaining and references for data alternative. Values of the levels and gamma-rays properties in the Adopted Levels, Gammas data sets are results of the analysis of corresponding values presented in "experimental" data sets. As an example, the mechanism of the forming the Adopted Levels, Gammas data set for ${ }^{60} \mathrm{Ni}$ is presented on Fig. 2. It must be pointed out that at this stage new physical information can appear already. For example, if one experiment gives spin values $1 / 2$ or $3 / 2$ for any level and another experiments gives $3 / 2$ or $5 / 2$ for the same level, evaluator assigns unambiguous spin value $3 / 2$ to this level, etc. 
Fig. 3 shows scheme of the using the data from the ENSDF for calculation of the subshell structure values:

- getting of both best nucleon pick-up and stripping reaction data from ENSDF in computerreadable form easily and quickly;

- getting of complete and reliable information on spins and parities of the final nuclei levels from the ENSDF Adopted Levels, Gammas data set;

- joint processing of the nucleon pick-up and stripping reaction data using the method described below.

It is very important to underline that important feature of the nucleon stripping and pick-up experiments is that total transferred momentum value $j$ cannot be measured as a rule with exception of experiments with polarized particles. But if the initial nucleus spin and parity are equal to $0^{+}$(all the even-even isotopes are of such case) this total transferred momentum value is equal to final nucleus level spin. So it is very important to have accurate, reliable and full data on spins of the isotopes.

\section{THE METHOD OF PUTTING NUCLEON PICK-UP AND STRIPPING EXPERIMENTS DATA IN ACCORDANCE TO EACH OTHER}

The methods of the extracting of the physical information from data of any experiment are based usually on using of relationships of a definite degree of generalization. If it is possible to connect data of two or more experiments using more general relations than those used for data extracting from experiments it means that one has a group of complementary experiments. Connecting each other of the complementary experimental data via more general relations can help to one to overcome certain limitation of information typical to the data obtained in ordinary way. Really it means a removing a systematical deviations caused by assumed model dependence from data analyzed jointly. The experiments of the nucleon pick-up and stripping reactions on the same initial nucleus present an example of the complementary pair concerns. The values of the nuclear levels spectroscopic factors can be obtained from these experiments by taking into account the DWBA normalization procedure and definite view of the nuclear potentials. As a result, absolute values of the spectroscopic values can differ by factor 2 and more. Besides, ignorance of some $j$ values mentioned above increases the systematical uncertainty significantly.

Meanwhile, one can connect sums of the spectroscopic factors values obtained from two types experiments under discussion via the quantum mechanics sum rules only /2/ without taking into account concrete type of nuclear interaction. It was shown $/ 2$ / that correctness of these sum rules is not less than $10 \%$ and consequently one can use these sum rules for getting new more precise 
normalization. In the framework of this approach the only one feature has meaning, namely that experimental cross-sections are proportional to spectroscopic factors values.

The main idea of the method of putting nucleon pick-up and stripping experiment data in accordance $/ 3$ / is to correct the experimental data so that following demands

$$
S_{n l j}^{+}+S_{n l j}^{-}=2 j+1
$$

would fill out for 3 single-particle orbits closest to Fermi energy, experimental data for which are presented with maximum of completeness,

$$
S_{n l j}^{+}+S_{n l j}^{-} \leq 2 j+1
$$

for the rest, and

$$
\left|\sum_{n l j} S_{n l j}^{-}-\sum_{n l j} S_{n l j}^{+}-N\right| \rightarrow 0
$$

for the all. Here $S_{n l j}^{\mp}$ are sums of the of the individual spectroscopic factor values $S_{n l j}^{\mp}\left(\mathrm{E}_{\mathrm{x}}\right)$ of the levels with energies $E_{x}$, upper signs + and - denote nucleon stripping and pick-up, correspondingly, summing in (3) is filled over valency and upper subshells in the first sum and over lower subshells in the second one, and $\mathrm{N}$ is a total number of corresponding nucleons (protons or neutrons) in a nucleus. Essence of the equation (3) is that a residual interaction does not change the total number in nucleus. To achieve these aims two freedom degrees are used:

- $\quad$ new normalization for experimental data is introduced $\left(S_{n l j}^{+}\left(\mathrm{E}_{\mathrm{x}}\right) \rightarrow \mathrm{n}^{+} S_{n l j}^{+}\left(\mathrm{E}_{\mathrm{x}}\right), S_{n l j}^{-}(\mathrm{Ex}) \rightarrow \mathrm{n}^{-}\right.$ $S_{n l j}^{-}(\mathrm{Ex})$

- all known information on spin of the final states is used, and all possibilities are investigated for states with unknown spins.

The codes ARES were developed on the base described procedures.

As a result, intervals for factors $\mathrm{n}+$ and $\mathrm{n}^{-}$and for $j$ values are determined. More realistic spectroscopic factors allow avoiding of discrepancies between various experimental data, as between various pick-up and various stripping data, as between pick-up and stripping data. Nucleon occupation probabilities of single-particle orbits

$$
N_{n l j}=\frac{\left[S_{n l j}^{-}+\left(2 j+1-S_{n l j}^{+}\right)\right]}{2(2 j+1)}
$$

and single-particle energies

$$
-E_{n l j}=\left(1-N_{n l j}\right)\left[B(A+1)-e_{n l j}^{+}\right\rfloor+N_{n l j}\left[B(A)+e_{n l j}^{-}\right\rfloor
$$


are determined on the base of improved spectroscopic factors. In (5) $B(A), B(A+1)$ are separation energy values of corresponding nucleon in target nucleus and in nucleus with one nucleon more, $e_{n l j}^{+}$, $e_{-l j}^{-}$are centroids of the spectroscopic factors distributions.

Such kind results were obtained for nuclei of ${ }^{40,42,44,46,48} \mathrm{Ca},{ }^{46,48,50} \mathrm{Ti},{ }^{50,52,54} \mathrm{Cr},{ }^{54,56,58} \mathrm{Fe}$, ${ }^{58,60,62,64} \mathrm{Ni},{ }^{64,66,68,70} \mathrm{Zn},{ }^{90,92,94,96} \mathrm{Zr},{ }^{116,118,120} \mathrm{Sn}$ both for neutron and proton orbits.

\section{IS ${ }^{96}$ ZR MAGIC NUCLEUS?}

Table 1 presents energy values of the first $2^{+}$states in ${ }^{90,92,94,96} \mathrm{Zr}$ isotopes. Maxima at $\mathrm{N}=50$ and $\mathrm{N}=56$ are seen very clearly. The $\mathrm{N}=50$ maximum has ordinary explanation because 50 is a wellknown magic number. But the $\mathrm{N}=56$ maximum demands a special explanation. Both neutron and proton subshells were a subjects of investigation from the point of view of one-nucleon transfer reaction data using the method described above. Neutron single-particle energies are presented on Fig. 4. With $\mathrm{N}$ increasing one can see dynamics of the $2 \mathrm{~d}_{5 / 2}$ subshell separation from upper shell so that in ${ }^{96} \mathrm{Zr}$ this subshell becomes look like as separate shell. Since in ${ }^{96} \mathrm{Zr}$ the orbit $2 \mathrm{~d}_{5 / 2}$ is closed, $\mathrm{N}=56$ becomes look like a magic number. It is necessary to note that analogous picture of the forming the separate shell $1 \mathrm{f}_{7 / 2}$ in calcium isotopes was obtained earlier/4/ with explanation of the $\mathrm{N}=28$ as a magic number (Fig.5). Correspondingly, two maxima at two magic numbers $\mathrm{N}=20$ and $\mathrm{N}=28$ one can observe in the first $2^{+}$states energy value dependencies on $\mathrm{N}$ in $\mathrm{Ca}$ isotopes (Table 2).

Table 3 presents results of our proton subshell investigation for $\mathrm{Zr}$ isotopes. The essential peculiarity is proton occupation probabilities of $\lg _{9 / 2}$ orbit in these isotopes. While these occupation probability values for ${ }^{90,92,94} \mathrm{Zr}$ are close to 0.1 (corresponds to 1 proton on the $1 \mathrm{~g}_{9 / 2}$ orbit), for ${ }^{96} \mathrm{Zr}$ this probability is exactly zero. This result presents an consequence of the final Y states spectroscopic factors distributions: while for ${ }^{89,91,93} \mathrm{Y}$ one can observe a transfer with $l=4, j=9 / 2$ and $\mathrm{c}^{2} \mathrm{~S}=0.9-1.3$ to levels with excitation energies $0.5-0.9 \mathrm{MeV}$, such transfers among the states of ${ }^{95} \mathrm{Y}$ are not observed at all. It means that the structure reconstruction takes place in ${ }^{96} \mathrm{Zr}$ so that this nucleus becomes more hard and, probably, more spherical, and residual collective energies are not sufficient to populate upper $1 \mathrm{~g}_{9 / 2}$ orbit. Such structure as known is corresponding to magic nuclei, and we can conclude that $\mathrm{N}=56$ is a magic number for $\mathrm{Z}=40$.

It is very important to pay attention that value $\mathrm{N}=56$ loses its magicity in pairs with another $\mathrm{Z}$, i.e. for $\mathrm{Mo}, \mathrm{Ru}$ isotopes, etc. This fact forces one to pay attention to relation between numbers 40 and 56. Let we note that 56 is $2 \times 28$, and 40 is $2 \times 20$ and imagine an analogy between ${ }^{96} \mathrm{Zr}$ and ${ }^{48} \mathrm{Ca}$ again. Indeed this analogy goes far, and if we consider data on decays of these nuclei we will see much more surprising coincidences. So, both ${ }^{48} \mathrm{Ca}$ and ${ }^{96} \mathrm{Zr}$ decay via $2 \beta^{-}$mode - quite rare decay, not more than 
$1 \%$ of all decays, and $\mathrm{T}_{1 / 2}\left({ }^{48} \mathrm{Ca}\right)=\left(4.2^{+3.3}{ }_{-1.3}\right) \times 10^{19}$ years $/ 5 /, \mathrm{T}_{1 / 2}\left({ }^{96} \mathrm{Zr}\right)=\left(2.1^{+0.8(\text { stat })}{ }_{-0.4(\text { stat })} \pm 0.2\right.$ (syst) $)$ $\times 10^{19}$ years /6/. It is really fantastic coincidence: one nucleus is twice as another, they both decay via the same rare mode, and half-life time of one is exactly twice as that for another.

All these facts can be explained if to suppose existence of additional interaction between closed $\mathrm{Z}=20$ and $\mathrm{N}=28$ structures in nuclei of ${ }^{48} \mathrm{Ca}$ and ${ }^{96} \mathrm{Zr}$. It is known that ${ }^{48} \mathrm{Ca}$ is neutron-rich but quite stable isotope. Interaction between proton $\mathrm{Z}=20$ and neutron $\mathrm{N}=28$ closed structures can be responsible for this specific stability. Connection between these structures can be destroyed with some probability giving $2 \beta^{-}$-decay of ${ }^{48} \mathrm{Ca}$ with appropriate half-life. In ${ }^{96} \mathrm{Zr}$ the same " $20-28$ " connections are presented twice, and therefore this nucleus $2 \beta^{-}$-decay must have half-life less by factor exactly 2 .

At least two schemes of connections can be proposed in framework of this consideration: the compound cluster model and the "nuclear crystal" model. The first model (Fig.6) supposes two levels of like-cluster structure interactions. The interaction $a$ connects 20 - and 28 - structures to ${ }^{48} \mathrm{Ca}$, and its destroying leads to $2 \beta^{-}$- decay with half-life $4.2 \times 10^{19}$ years. The interaction $b$ connects "clusters" ${ }^{48} \mathrm{Ca}$ to nucleus of ${ }^{96} \mathrm{Zr}$. Interaction $b$ is supposed to be stronger than $a$, and destroying one of two $a$ interactions leads to destruction of the whole system and to $2 \beta^{-}$-decay with total half-life $2.1 \times 10^{19}$ years. The second model supposes two-valent connection between 20- and 28- structures in nucleus of ${ }^{48} \mathrm{Ca} /$ Fig.7a/. Nucleus of ${ }^{96} \mathrm{Zr}$ is constructed from these two-valent structures like a molecule /Fig.7b/, and since in both cases of ${ }^{48} \mathrm{Ca}$ and ${ }^{96} \mathrm{Zr}$ we have a systems with additional hardness, that could be characterized as "nuclear crystal".

\section{PROTON SUBHELLS IN CA ISOTOPES}

Nucleon occupation probabilities and single-particle energies of proton orbits in ${ }^{40,42,44,46,48} \mathrm{Ca}$ are presented in Table 4. Fig. 8 presents single-particle energies of the proton orbits in the ${ }^{40,42,44,46,48} \mathrm{Ca}$ isotopes. One can observe the inversion of the $1 \mathrm{~d}_{3 / 2}$ and $2 \mathrm{~s}_{1 / 2}$ orbits in ${ }^{48} \mathrm{Ca}$ isotope. This peculiarity explains irregularities in spin-parity values of ground states of nuclei of $\mathrm{K}:{ }^{39,41,43,45} \mathrm{~K}$ ground states have a $\mathrm{J}^{\pi}=3 / 2^{+}$, but ${ }^{47} \mathrm{~K}-1 / 2^{+}$. To describe features of the proton orbits placement in ${ }^{48} \mathrm{Ca}$, in particular, $1 d_{3 / 2}-2 s_{1 / 2}$-inversion, it was supposed that spin-orbit splitting decreases in this nucleus (see Fig. 9). This hypothesis was tested in the dispersion optical model calculations for ${ }^{40,42,44,46,48} \mathrm{Ca} / 7 /$ and it was cleared that this assumption allows to describe correctly this inversion.

\section{CONCLUSIONS}

On the base of international nuclear spectroscopy data bank ENSDF as power tool for investigation of the single-particle structure of nuclei very rich new single-particle information (single- 
particle energy and nucleon occupation probability values for various orbits) has been obtained for many nuclei.

The most important conclusions could be done are the following

1) Features of the first $J^{\pi}=2^{+}$level energy values in nuclei $\mathrm{Zr}$ can be explained in a framework of shell model approach. A forming of the $2 \mathrm{~d}_{5 / 2}$ shell in ${ }^{96} \mathrm{Zr}$ (like $1 \mathrm{f}_{7 / 2}$ in ${ }^{48} \mathrm{Ca}$ ) is discovered, so $\mathrm{N}=$ 56 becomes look like a magic number for $Z=40$. To explain some coincidences in decay properties of ${ }^{48} \mathrm{Ca}$ and ${ }^{96} \mathrm{Zr}$ additional interaction between closed 20 - and 28 -structure was proposed.

2) Irregularities of the spin values in nuclei of $K$ can be explained in a framework of shell model approach by inversion of the $1 \mathrm{~d}_{3 / 2}$ and $2 \mathrm{~s}_{1 / 2}$ proton orbits.

\section{ACKNOWLEDGEMENTS}

Authors acknowledged the fruitful discussions and help of Prof. E.A.Romanovsky and Dr. O.V.Bespalova.

Research was carried out at the MSU SINP Department of Electromagnetic Processes and Atomic Nuclei Interaction and supported in part by the President of Russia grant N SS-1619.2003.2 and the RBFR grant N 03-07-90431.

\section{REFERENCES}

1. T.W.Burrows, Nucl. Instr. and Meth. in Phys. Res. A286 (1990) 5953 .

2. C.F.Clement, Nucl.Phys. A213 (1973) 469.

3. I.N. Boboshin, V.V.Varlamov, B.S.Ishkhanov and I.M.Kapitonov, Nucl. Phys. A496 (1989) 93.

4. O.V.Bespalova, I.N.Boboshin, V.V.Varlamov et.al. Izv.Akad.Nauk. (to be published).

5. V. B. Brudanin, N. I. Rukhadze, Ch. Briançon et.al. Phys. Lett. B 495 (2000) 63.

6. R. Arnold, C. Augier, J. Baker et.al. Nucl.Phys. A658 (1999) 299.

7. O.V.Bespalova, I.N.Boboshin, V.V.Varlamov et.al. Yad.Fiz 66 (2003) 1 .

Table1.

Energies of the first $2^{+}$states of even-even nuclei of $Z$ r.

\begin{tabular}{|l|c|c|c|c|}
\hline Nucleus & ${ }^{90} \mathrm{Zr}$ & ${ }^{92} \mathrm{Zr}$ & ${ }^{94} \mathrm{Zr}$ & ${ }^{96} \mathrm{Zr}$ \\
\hline Number of neutrons N & 50 & 52 & 54 & 56 \\
\hline $\mathrm{E}\left(2^{+}, 1\right)(\mathrm{keV})$ & 2186.50 & 934.48 & 918.75 & 1750.47 \\
\hline
\end{tabular}


Table 2 .

Energies of the first $2+$ states of the even-even nuclei of $\mathrm{Ca}$.

\begin{tabular}{|l|c|c|c|c|c|}
\hline Nucleus & ${ }^{40} \mathrm{Ca}$ & ${ }^{42} \mathrm{Ca}$ & ${ }^{44} \mathrm{Ca}$ & ${ }^{46} \mathrm{Ca}$ & ${ }^{48} \mathrm{Ca}$ \\
\hline Number of neutrons $\mathrm{N}$ & 20 & 22 & 24 & 26 & 28 \\
\hline $\mathrm{E}(2+, 1)(\mathrm{keV})$ & 3904.50 & 1524.61 & 1157.02 & 1346.00 & 3831.72 \\
\hline
\end{tabular}

Table 3 .

Nucleon occupation probabilities $\mathrm{N}_{\mathrm{nlj}}$ and single-particle energies $-\mathrm{E}_{\text {nlj }}(\mathrm{MeV})$ of proton orbits in nuclei of ${ }^{90,92,94,96} \mathrm{Zr}$

\begin{tabular}{|l|c|c|c|c|c|}
\hline $\mathrm{Nlj}$ & $\mathrm{N}_{\mathrm{nlj}}-\mathrm{E}_{\mathrm{nlj}}$ & ${ }^{90} \mathrm{Zr}$ & ${ }^{92} \mathrm{Zr}$ & ${ }^{94} \mathrm{Zr}$ & ${ }^{96} \mathrm{Zr}$ \\
\hline $1 \mathrm{~g}_{9 / 2}$ & $\mathrm{~N}_{\mathrm{nlj}}$ & $0.06(5)$ & $0.08(5)$ & $0.09(5)$ & $0.00(0)$ \\
& $-\mathrm{E}_{\mathrm{nlj}}$ & $5.41(54)$ & $4.98(142)$ & $6.74(80)$ & $7.48(750$ \\
\hline $2 \mathrm{p}_{1 / 2}$ & $\mathrm{~N}_{\mathrm{nlj}}$ & $0.58(5)$ & $0.49(3)$ & $0.75(5)$ & $0.81(5)$ \\
& $-\mathrm{E}_{\mathrm{nlj}}$ & $6.97(70)$ & $7.66(77)$ & $9.37(94)$ & $10.59(106)$ \\
\hline $1 \mathrm{f}_{5 / 2}$ & $\mathrm{~N}_{\mathrm{nlj}}$ & $1.00(2)$ & $1.00(2)$ & $1.00(2)$ & $0.94(5)$ \\
& $-\mathrm{E}_{\mathrm{nlj}}$ & $10.37(110)$ & $10.93(110)$ & $11.49(115)$ & $12.17(122)$ \\
\hline $2 \mathrm{p}_{3 / 2}$ & $\mathrm{~N}_{\mathrm{nlj}}$ & - & - & $0.87(5)$ & - \\
& $-\mathrm{E}_{\mathrm{nlj}}$ & - & - & $11.11(112)$ & - \\
\hline
\end{tabular}

Table 4.

Nucleon occupation probabilities $\mathrm{N}_{\mathrm{nlj}}$ (upper)

and single-particle energies $-\mathrm{E}_{\mathrm{nlj}}(\mathrm{MeV})$ (lower) of proton subshells in nuclei of ${ }^{40.42 .44 .46 .48} \mathrm{Ca}$

\begin{tabular}{|l|c|c|c|c|c|}
\hline & ${ }^{40} \mathrm{Ca}$ & ${ }^{42} \mathrm{Ca}$ & ${ }^{44} \mathrm{Ca}$ & ${ }^{46} \mathrm{Ca}$ & ${ }^{48} \mathrm{Ca}$ \\
\hline \multirow{3}{*}{$1 \mathrm{f}_{5 / 2}$} & - & - & - & - & 0.00 \\
\cline { 2 - 6 } & - & - & - & - & $3817(128)$ \\
\hline \multirow{2}{*}{$2 \mathrm{p}_{1 / 2}$} & 0.00 & 0.00 & - & - & $0.01(1)$ \\
\cline { 2 - 6 } & $-2378(28)$ & $<129$ & - & - & $2353(645)$ \\
\hline \multirow{2}{*}{$2 \mathrm{p}_{3 / 2}$} & $0.09(0.02)$ & $0.02(0.01)$ & $0.05(2)$ & - & $0.01(1)$ \\
\cline { 2 - 6 } & $732(289)$ & $1300(132)$ & $4989(129)$ & - & $3954(368)$ \\
\hline \multirow{2}{*}{$1 \mathrm{f}_{7 / 2}$} & $0.06(2)$ & $0.08(3)$ & $0.13(3)$ & $0.02(2)$ & $0.02(2)$ \\
\cline { 2 - 6 } & $1669(152)$ & $4092(190)$ & $7681(174)$ & $7892(596)$ & $8626(514)$ \\
\hline \multirow{2}{*}{$1 \mathrm{~d}_{3 / 2}$} & $0.97(3)$ & $0.76(3)$ & $0.72(1)$ & $0.94(3)$ & $0.94(5)$ \\
\cline { 2 - 6 } & $9523(1195)$ & $10027(1122)$ & $10813(8)$ & $13533(306)$ & $15960(600)$ \\
\hline $2 \mathrm{~s}_{1 / 2}$ & 1.00 & 0.90 & 0.77 & $0.93(1)$ & $0.84(5)$ \\
\cline { 2 - 6 } & 10942 & $>11286$ & 11395 & $13937(58)$ & $14415(655)$ \\
\hline \multirow{2}{*}{$1 \mathrm{~d}_{5 / 2}$} & 0.96 & - & - & - & - \\
\cline { 2 - 6 } & 14321.37 & - & - & - & - \\
\hline
\end{tabular}




\section{FIGURE CAPTURES}

Fig.1. Organization of the ENSDF.

Fig.2. Scheme of the forming the Adopted Levels. Gammas data set in ENSDF.

Fig.3. An example of the ENSDF data using for obtaining the single-particle nuclear structure parameters.

Fig.4. Neutron subshells of nuclei of ${ }^{90.92 .94 .96} \mathrm{Zr}$.

Fig.5. Neutron subshells of ${ }^{40.42 .44 .46 .48} \mathrm{Ca}$.

Fig.6. Compound cluster model of ${ }^{96} \mathrm{Zr}$.

Fig.7. 2-valent connection model:

$$
\text { a - }{ }^{48} \mathrm{Ca} \text {; }
$$

Fig.8. On explanation of the inversion of the $1 \mathrm{~d}_{3 / 2}$ and $2 \mathrm{~s}_{1 / 2}$ proton orbits in ${ }^{48} \mathrm{Ca}$. 
EVALUATED NUCLEAR STRUCTURE DATA FILE

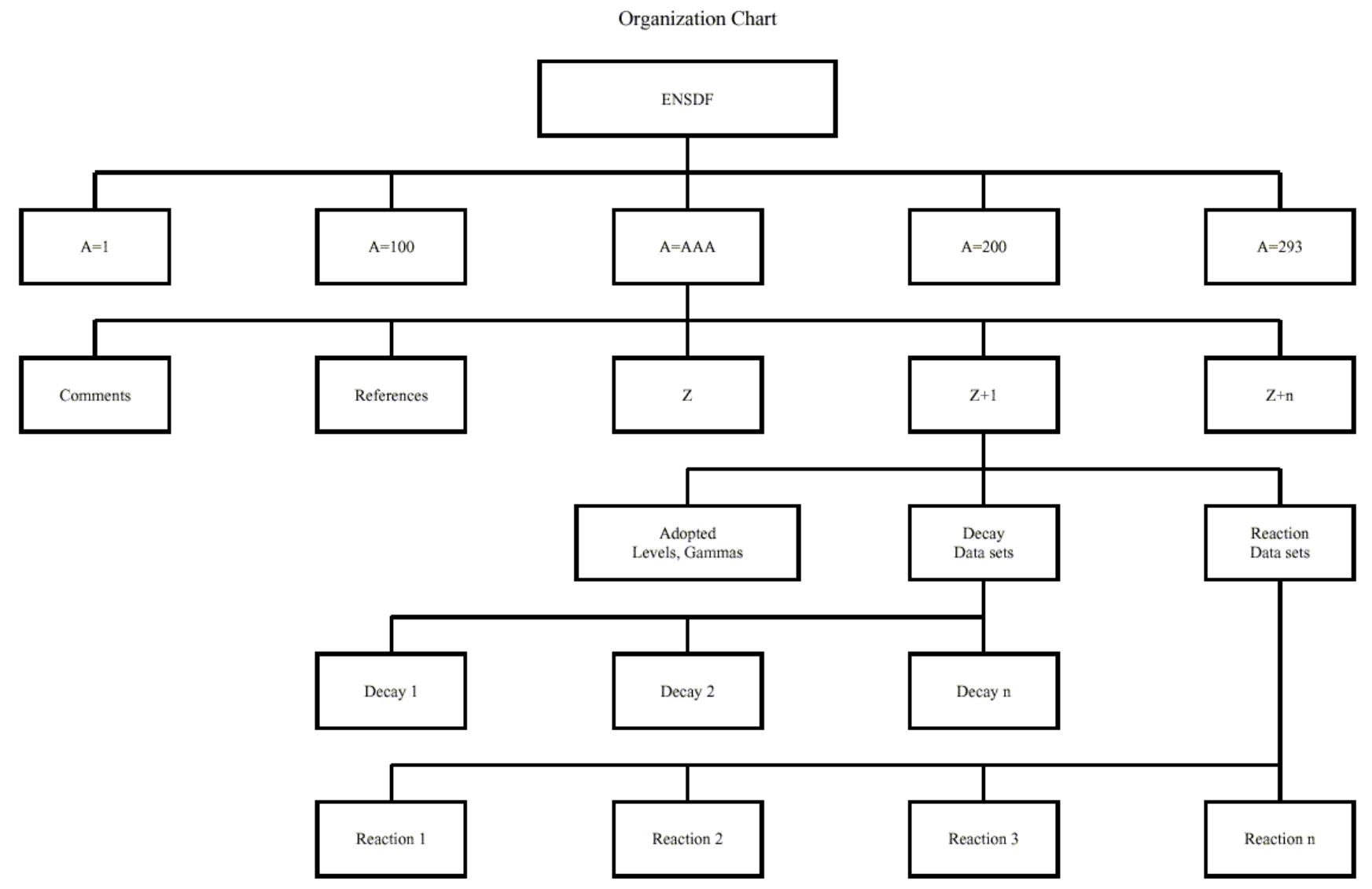

Fig.1. Organization of the ENSDF. 
The data sets for nusleus of ${ }^{59} \mathrm{Ni}$ from ENSDF

\begin{tabular}{|c|c|c|c|}
\hline & UCL. ${ }^{\mathrm{N}}$ & THE DATA SET & $\begin{array}{l}\text { NUMBER } \\
\text { OF } \\
\text { RECORDS }\end{array}$ \\
\hline 1 & $59 \mathrm{NI}$ & ADOPTED LEVELS, GAMMAS & 1149 \\
\hline 2 & $59 \mathrm{NI}$ & 59CU EC DECAY & 109 \\
\hline 3 & $59 \mathrm{NI}$ & $50 \mathrm{CR}(12 \mathrm{C}, 2 \mathrm{PNG})$ & 74 \\
\hline 4 & $59 \mathrm{NI}$ & $56 \mathrm{FE}(\mathrm{A}, \mathrm{NG})$ & 161 \\
\hline 5 & $59 \mathrm{NI}$ & $56 \mathrm{FE}(6 \mathrm{LI}, \mathrm{T})$ & 17 \\
\hline 6 & $59 \mathrm{NI}$ & 58NI(N,G), (POL N,G) E=THERMAL & 372 \\
\hline 7 & $59 \mathrm{NI}$ & $58 \mathrm{NI}(\mathrm{N}, \mathrm{G}) \mathrm{E}=2-90 \mathrm{KEV}$ & 53 \\
\hline 8 & $59 \mathrm{NI}$ & 58NI(D,P), (POL D,P) & 277 \\
\hline 9 & $59 \mathrm{NI}$ & 58NI(POL T,D) & 16 \\
\hline 10 & $59 \mathrm{NI}$ & $58 \mathrm{NI}(3 \mathrm{HE}, 2 \mathrm{PG})$ & 226 \\
\hline 11 & $59 \mathrm{NI}$ & $58 \mathrm{NI}(\mathrm{A}, 3 \mathrm{HE})$ & 33 \\
\hline 12 & $59 \mathrm{NI}$ & 58NI(POL 7LI,6LI) & 12 \\
\hline 13 & $59 \mathrm{NI}$ & $58 \mathrm{NI}(14 \mathrm{C}, 13 \mathrm{C})$ & 27 \\
\hline 14 & $59 \mathrm{NI}$ & $58 \mathrm{NI}(16 \mathrm{O}, 15 \mathrm{O})$ & 12 \\
\hline 15 & $59 \mathrm{NI}$ & $59 \mathrm{CO}(\mathrm{P}, \mathrm{N})$ & 55 \\
\hline 16 & $59 \mathrm{NI}$ & $59 \mathrm{CO}(\mathrm{P}, \mathrm{NG})$ & 148 \\
\hline 17 & $59 \mathrm{NI}$ & 60NI(P,D), (POL P,D) & 100 \\
\hline 18 & $59 \mathrm{NI}$ & $60 \mathrm{NI}(\mathrm{D}, \mathrm{T}),(\mathrm{POL} \mathrm{D}, \mathrm{T})$ & 27 \\
\hline 19 & $59 \mathrm{NI}$ & $60 \mathrm{NI}(3 \mathrm{HE}, \mathrm{A})$ & 82 \\
\hline 20 & $59 \mathrm{NI}$ & $61 \mathrm{NI}(\mathrm{P}, \mathrm{T})$ & 5 \\
\hline
\end{tabular}
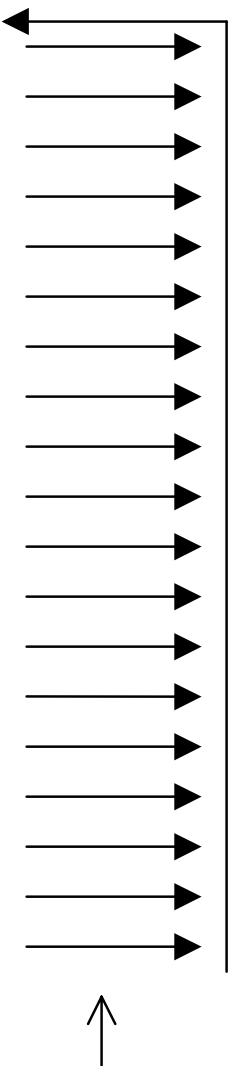

DATA ON LEVELS AND GAMMA-

TRANSITIONS IN 59NI

(energies, spins, parities, isospins, momenta, intensities, multipolarities, etc.)

Fig.2. Scheme of the forming the Adopted Levels. Gammas data set in ENSDF. 


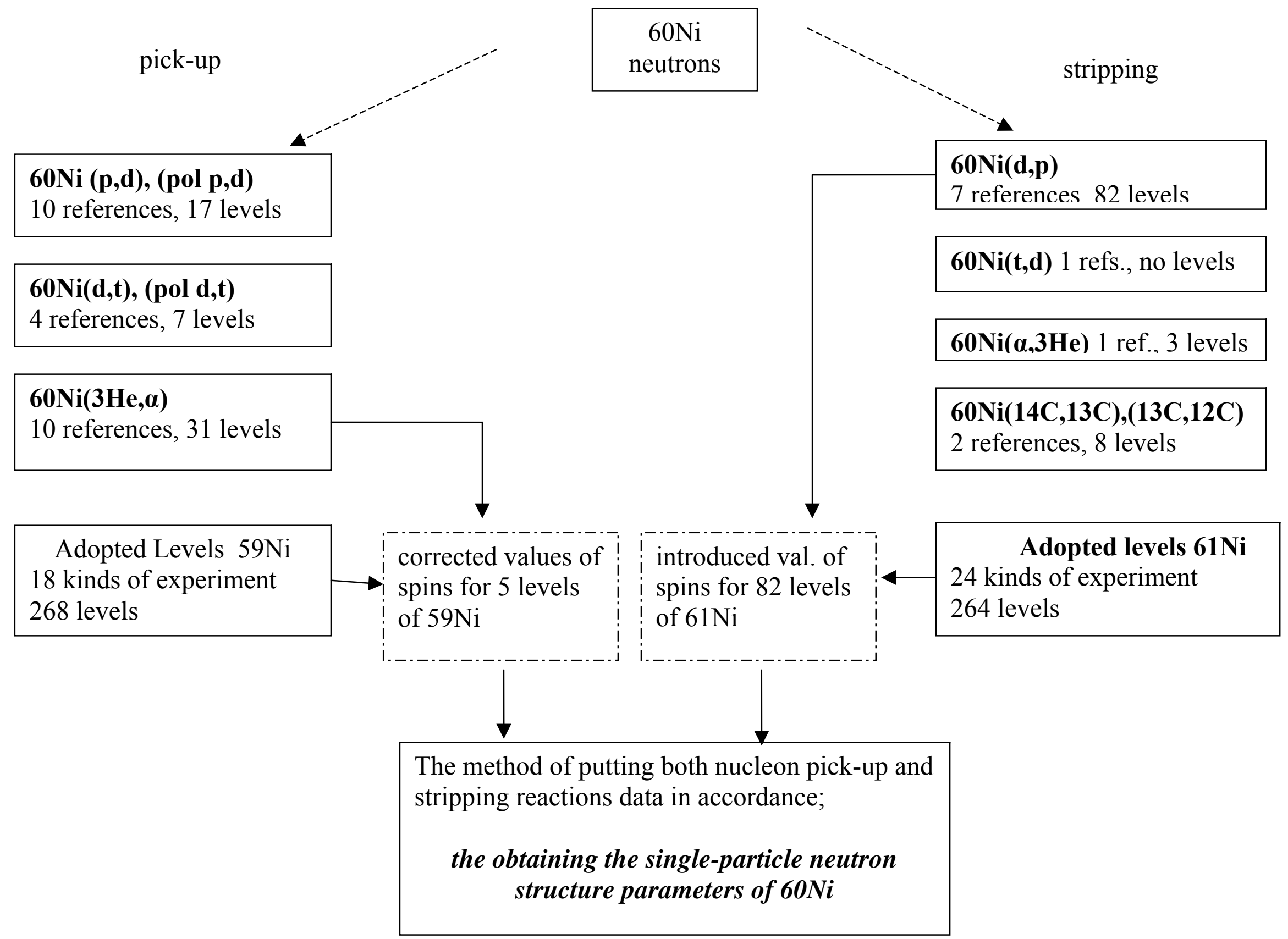




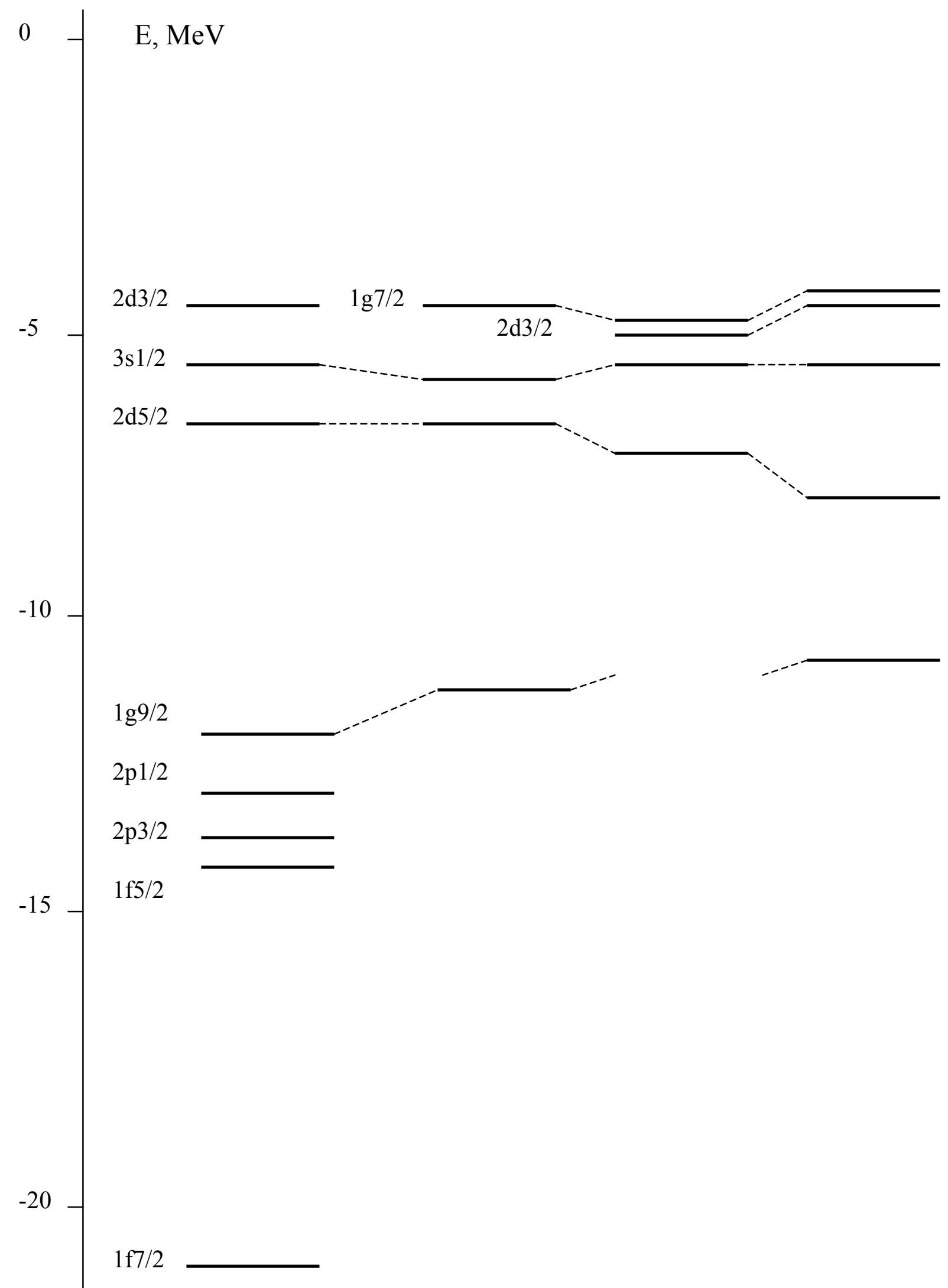

Fig.4. Neutron subshells of nuclei of ${ }^{90.92 .94 .96} \mathrm{Zr}$. 


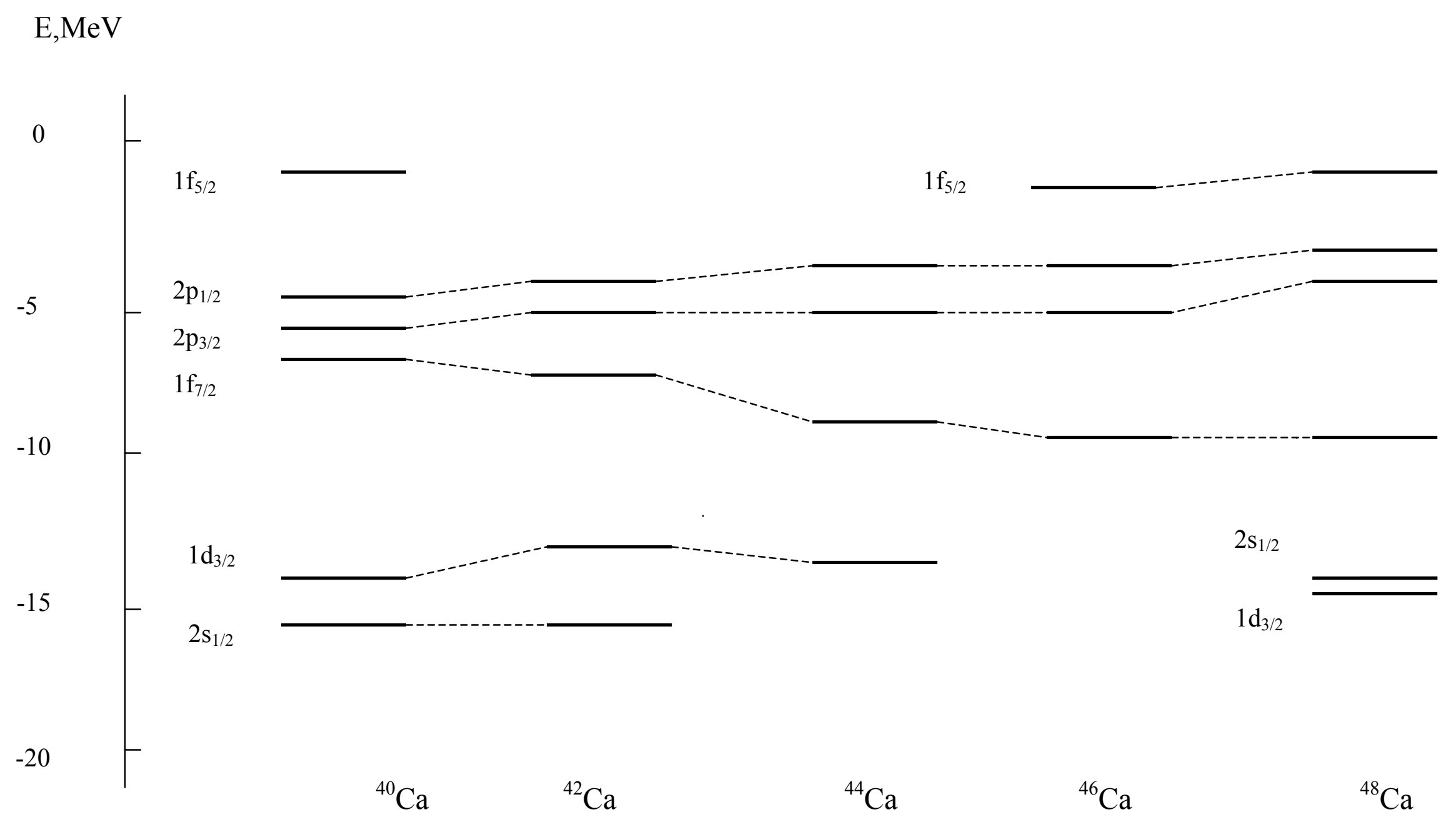

Fig.5. Neutron subshells of ${ }^{40.42 .44 .46 .48} \mathrm{Ca}$. 


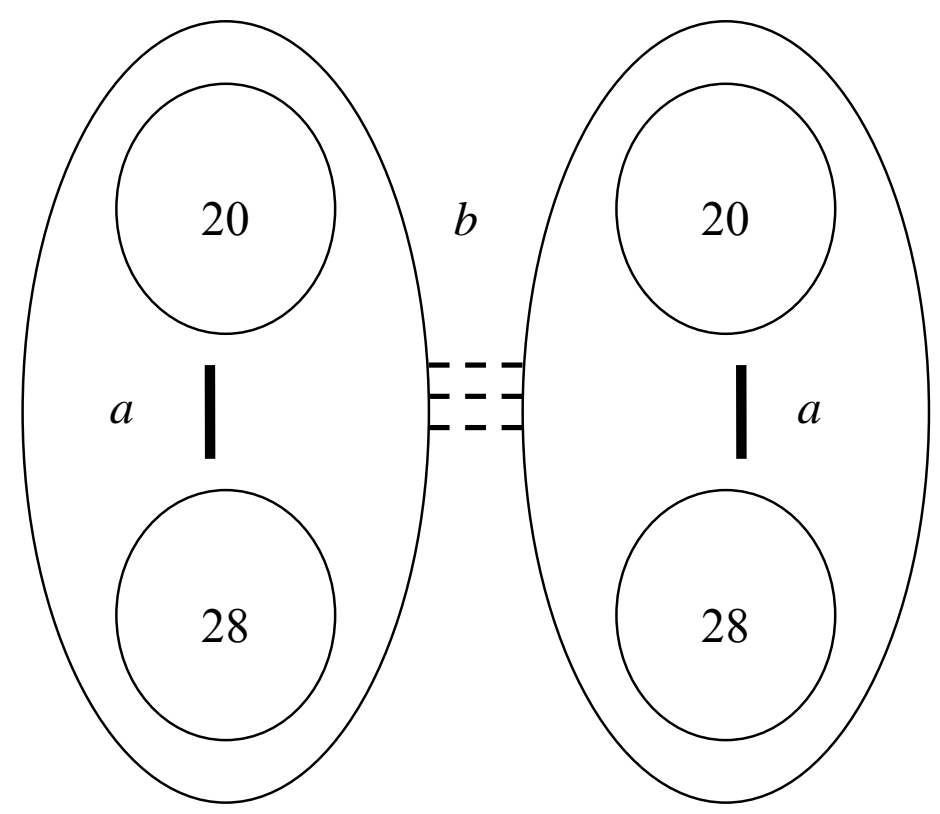

Fig.6. Compound cluster model of ${ }^{96} \mathrm{Zr}$. 
a
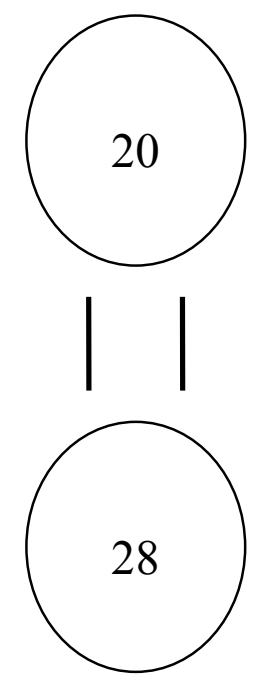

b

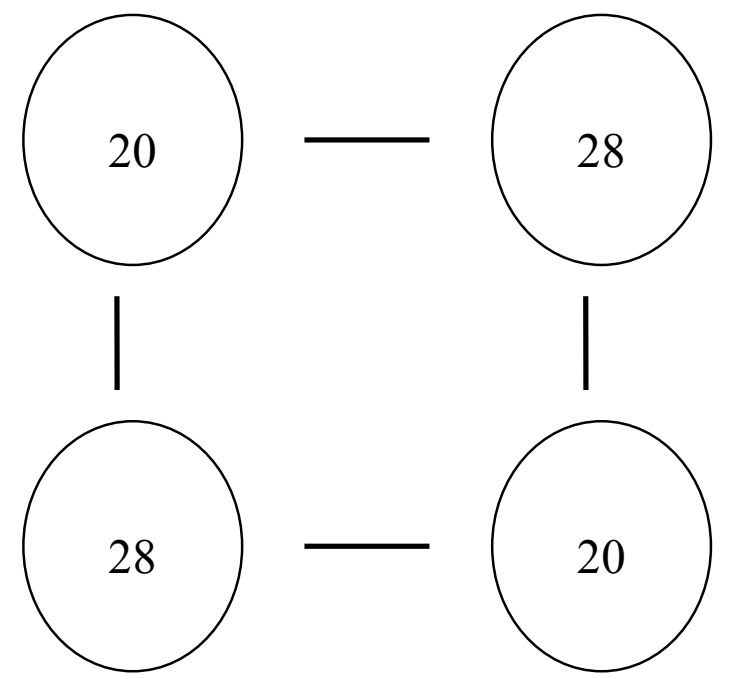

Fig.7. 2-valent connection model:

$\mathrm{a}-{ }^{48} \mathrm{Ca}$;

b $-{ }^{96} \mathrm{Zr}$. 
$1 d_{3 / 2}$
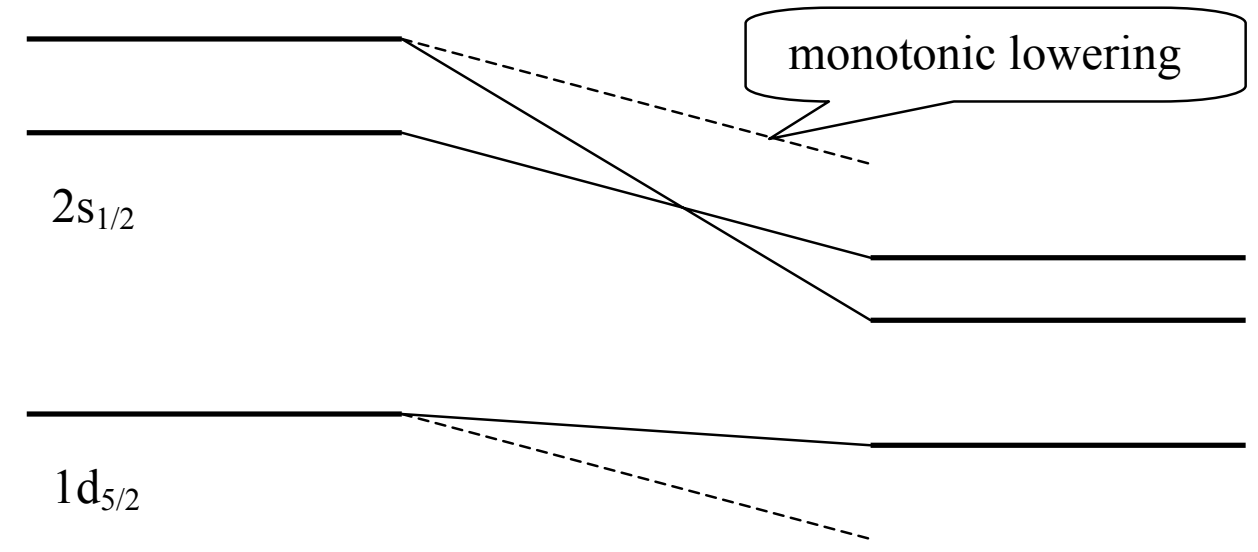

Fig.8. On explanation of the inversion of the $1 \mathrm{~d}_{3 / 2}$ and $2 \mathrm{~s}_{1 / 2}$ proton orbits in ${ }^{48} \mathrm{Ca}$. 